\title{
Laboreal
}

Volume $10 \mathrm{~N}^{\circ} 2$ | 2014

Análise ergonómica do trabalho e formação - Parte II

\section{La transmisión profesional : procesos de elaboración de interacciones formativas en situación de trabajo. Una investigación del personal de cuidados en salud de un Centro Hospitalario Universitario}

A transmissão profissional : processo de elaboração de interações formativas em situação de trabalho : uma pesquisa realizada junto à equipe de cuidados em saúde de um Centro Hospitalar Universitário

La transmission professionnelle : processus d'élaboration d'interactions formatives en situation de travail : une recherche auprès de personnels soignants dans un Centre Hospitalier Universitaire

Transfer of professional skills: processes of elaboration of formative interactions in work situations: a research focused on the nursing staff in a University Hospital

Jeanne Thébault

\section{OpenEdition}

Journals

Edición electrónica

URL: http://journals.openedition.org/laboreal/4986

DOI: $10.4000 /$ laboreal.4986

ISSN: 1646-5237

Editor

Universidade do Porto

Referencia electrónica

Jeanne Thébault, « La transmisión profesional : procesos de elaboración de interacciones formativas en situación de trabajo. Una investigación del personal de cuidados en salud de un Centro

Hospitalario Universitario », Laboreal [En línea], Volume 10 №2 | 2014, Publicado el 01 diciembre 2014, consultado el 24 septiembre 2020. URL : http://journals.openedition.org/laboreal/4986 ; DOI : https:// doi.org/10.4000/laboreal.4986

Este documento fue generado automáticamente el 24 septiembre 2020.

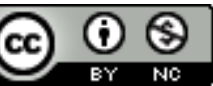

Laboreal está licenciado com uma Licença Creative Commons - Atribuição-NãoComercial 4.0 Internacional. 


\section{La transmisión profesional : procesos de elaboración de interacciones formativas en situación de trabajo. Una investigación del personal de cuidados en salud de un Centro Hospitalario Universitario}

A transmissão profissional : processo de elaboração de interações formativas em situação de trabalho: uma pesquisa realizada junto à equipe de cuidados em saúde de um Centro Hospitalar Universitário

La transmission professionnelle : processus d'élaboration d'interactions formatives en situation de travail : une recherche auprès de personnels soignants dans un Centre Hospitalier Universitaire Transfer of professional skills: processes of elaboration of formative interactions in work situations: a research focused on the nursing staff in a University Hospital

Jeanne Thébault

REFERENCIA

Thébault, J. (2013). La transmission professionnelle : processus d'élaboration d'interactions formatives en situation de travail. Une recherche auprès de personnels 
soignants dans un Centre Hospitalier Universitaire. Thèse de doctorat en Ergonomie, CNAM/CEE-CREAPT, Paris.

\section{NOTA DEL EDITOR}

http://dx.doi.org/10.15667/laborealx0214jt

Manuscrito recibido en : Octubre /2014

Aceptado tras peritaje : Noviembre /2014

\section{La transmisión profesional en el centro de los desafíos de las evoluciones demográficas y del trabajo}

1 Si bien la temática de la transmisión de saberes profesionales en situaciones de trabajo no es novedosa, hoy se cristalizan alrededor de ella importantes preocupaciones, tanto en lo que respecta a las evoluciones del trabajo, así como de las evoluciones demográficas (Volkoff, 2011). El desafío mayor para las empresas consiste así en la creación de las condiciones para una circulación "flexible» de los saberes profesionales en su interior. Los establecimientos hospitalarios franceses están igualmente implicados y se encuentran afectados por profundas restructuraciones asociadas a las transformaciones políticas, sociales, económicas y de gestión que atraviesa el sector de la salud y su entorno (Gheorghiu, Guillemot, \& Moatty, 2009). La transmisión de saberes profesionales debe realizarse entonces en un contexto móvil que desestabiliza las prácticas del personal de cuidado de pacientes. Tal contexto convoca a examinar de más cercana las condiciones de realización de la transmisión profesional y sus posibilidades de despliegue.

2 La " transmisión profesional » se plantea aquí con una acepción más amplia que aquella referida a los dispositivos institucionales, designados bajo el término de "tutorado ». Se aborda como una actividad en toda su expresión, realizada en, por y para la actividad del trabajo, mediando a través de otro, las dimensiones de la práctica profesional. Se define como "el conjunto de los intercambios realizados en razón de las prácticas profesionales, durante la realización del trabajo, entre diferente individuos, trayectorias, experiencias y antigüedades. Dichos intercambios se encuentran marcados por las asimetrías que esta diversidad implica entre esos individuos » (Thébault, 2013, p 28). Esta investigación nos ha permitido construir un modelo en términos de " procesos de elaboración de interacciones formativas en situación de trabajo ». Las interacciones formativas se apoyan sobre tres componentes mayores, articulados entre ellos, formando una dinámica de conjunto : la co-construcción de una relación entre los protagonistas, la conciliación entre las actividades de transmisión y de producción y la combinación de saberes profesionales.

\section{Un camino metodológico con varias facetas}

Nuestra investigación se desarrolló en un Centro Hospitalario Universitario (CHU), a partir de un enfoque metodológico que considera de modo combinado métodos 
cuantitativos demográficos del trabajo (Molinié, \& Volkoff, 2002), y métodos cualitativos en ergonomía. Desde punto de vista de estos últimos, han sido particularmente investigados tres servicios y contrastados desde punto de vista de su especialidad médica, de su organización, de las características de los pacientes y de la composición de sus equipos (Cirugía cardíaca, Reumatología, Cuidados y Readaptación).

4 Fueron observados sistemáticamente doce turnos (enteros o parciales), enfocándose en las interacciones formativas. Estas observaciones fueron combinadas, en la medida de lo posible, con entrevistas, auto-confrontaciones o discusiones, realizadas principalmente con supervisoras enfermeras y técnicos paramédicos. Esas situaciones consideran diferentes formas de recepción de los recién llegados : siete situaciones de supervisión de estudiantes, seis situaciones de recepción de nuevos miembros asalariados en el servicio, una situación de recepción de personal externo al servicio (equipo de reemplazo para el conjunto del $\mathrm{CHU}$ ) y una situación de co-presencia entre personal permanente del servicio y personal compartido con otras unidades del servicio. Es de notar que, entre esas situaciones, seis de entre ellas combinan la acogida simultánea de varios recién llegados. Por último, esos elementos fueron completados por tres sesiones de "talleres reflexivos", realizados con estudiantes de enfermería de último año, en base a elementos previamente analizados.

\section{Las interacciones formativas en el trabajo : tres componentes articulados entre ellos}

5 El modelo de transmisión profesional acá propuesto se basa en una combinación de elementos provenientes de la literatura y del conjunto de los análisis de los datos de campo. Cada una de las situaciones observadas y los comentarios asociados, contribuyen a la caracterización de las diferentes dimensiones que se consideraron en la investigación. Antes de presentar los 3 componentes, comenzaremos por una introducción al contexto en el cual éstos fueron identificados.

\subsection{La transmisión profesional : una actividad bajo exigencias}

6 Un CHU tiene como misión, entre otras cosas, acoger, supervisar y proveer formación inicial y continua al personal de cuidados de salud. Este tipo de establecimiento público conlleva una doble lógica: de producción de cuidados de salud y de formación. Dos dispositivos institucionales de formación y de inserción ya se encuentran presentes: por una parte, la acogida de estudiantes en formación para la realización de sus prácticas profesionales, por otra, un período de dos semanas de "doblaje », de un nuevo colega con un profesional permanente del servicio. No obstante, al observar de cerca, se constata que esos dispositivos producen un escaso encuadramiento, dejando la gestión de la transmisión a la discreción de los equipos de salud.

7 Nuestro análisis de las condiciones de acogida et de supervisión dan cuenta de una intensificación de las exigencias temporales y de una inestabilidad de los equipos : la llegada de un nuevo colega no es siempre un evento anticipado por él, ni por el equipo que lo descubre recién al inicio del turno ; las exigencias del servicio pueden acortar el tiempo de doblaje, conduciendo al recién llegado a hacerse rápidamente cargo solo de una parte de las tareas, pudiendo este encontrarse además con un personal poco experimentado o ser derivado de un miembro del equipo a otro. Del mismo modo, es 
posible encontrar situaciones en las que un miembro del equipo puede tener que recibir nuevos miembros en forma simultánea; que algunos recién llegados pueden tener conocimiento previo del tipo de cuidados a brindar, de los pacientes o del material gracias a sus experiencias anteriores. En contraste, algunos miembros "antiguos" del equipo pueden encontrarse en situación de "novatos", cuando su servicio eventualmente cambia de especialidad, o cuando por efecto de sus diferentes asignaciones, las estadías no han sido lo suficientemente prolongadas en el servicio en cuestión. Además, es de considerar que la urgencia de una atención puede interrumpir o modificar las modalidades de transmisión ; el tiempo destinado a la supervisión de un recién llegado puede reducir la posibilidad de ayudar a otros colegas o puede implicar trasladar tareas al siguiente equipo en turno. Por último, la aplicación de un examen (para los estudiantes), puede perturbar el desarrollo de las curaciones que deben realizar, cambiando la organización del trabajo, generando problemas de trazabilidad, errores y eventuales olvidos o la imposibilidad de acceso a ciertos locales. De este modo, el carácter discrecional de esta tarea necesita de parte de los equipos de salud una fuerte inversión, tanto para crear las modalidades de interacciones formativas, como para ejecutarlas.

\subsection{Integrar la transmisión en la actividad de trabajo}

8 La transmisión se realiza durante la actividad de producción, compartiendo el mismo tipo de exigencias. Los protagonistas deben elaborar medios de arbitraje para conciliar lo mejor posible las actividades de producción y de transmisión. En contexto, los supervisores (más que los recién llegados) intentan construir, agenciar "espacios » (momento, lugar, etc.) para realizar la transmisión, en función de los márgenes de maniobra que disponen, pero también en función de aquellas que son capaces de construir individual y colectivamente. Tres formas de situaciones fueron identificadas en razón de las exigencias presentes: "puesta entre paréntesis", "tomar la oportunidad » y “ más allá del binomio ».

En las situaciones más exigentes (por ej. varios recién llegados presentes al mismo tiempo, reprogramación permanente de las tareas de cuidados, etc.) la transmisión será “puesta entre paréntesis ». Los márgenes de maniobra para conciliar actividades de transmisión y de trabajo son acotadas y la supervisión no es capaz de generarlos. La transmisión es entonces desplazada en prioridad con respecto a los objetivos de producción (el cuidado de los pacientes).

10 En las situaciones donde las exigencias son menos fuertes, las supervisoras "toman las oportunidades" que se presentan. En ese caso, las interacciones formativas se enriquecen, se desarrollan pruebas de conocimientos, ejercicios o discusiones colectivas. Las supervisoras tratan de generarse márgenes de maniobra para que la transmisión se desarrolle en buenas condiciones. El arreglo de esas condiciones no es sin embargo sin costo para las supervisoras, ya que incluso si los nuevos miembros del equipo se encuentran en condiciones de realizar solos algunas curaciones, las supervisoras deben permanecer atentas a lo que realizan, ya que el conjunto de atenciones se encuentran bajo su responsabilidad.

11 Por último, en las situaciones que calificamos " más allá del binomio ", es el colectivo el que toma a cargo la conciliación entre producción y transmisión, gestionando una para favorecer la otra. En ciertas situaciones, la supervisión de un estudiante puede 
transformarse en un espacio de discusión al interior del colectivo (intra e inter oficio) o de aprendizajes mutuos, y entre todos participar en mutualizaciones de las prácticas.

\subsection{Co-construir una relación}

12 Las interacciones formativas implican, al menos, dos actores. Su elaboración determina evidentemente los saberes profesionales abordados, pero tiene igualmente el propósito de formatear la propia relación. Esta noción de "relación " permite insistir sobre la dimensión temporal de las interacciones, más allá de un cara a cara instantáneo. Visualizar la co-construcción de una relación en elaboración de las interacciones formativas permite también tener en cuenta la actividad de cada uno de los protagonistas colocando el acento sobre la igualdad de sus intervenciones, mientras que, por otra parte, permite poner el acento sobre la componente colectiva de la propia actividad de construcción de la relación entre ellos.

De esta manera, el desarrollo del periodo de acogida y supervisión, ofrece a uno y a otro numerosas ocasiones de mostrar sus propios recursos y sus propias expectativas, evaluar aquellas del protagonista, de proponer más o menos explícitamente inflexiones en esta actividad común. Así, cada uno comprometido en la construcción de la relación, muestra lo que sabe o no sabe, cuestiona al otro, lo que permite a los protagonistas de “ajustarse ». Desde una primera mirada, este ajuste se apoya sobre los conocimientos que cada uno adquiere en relación al funcionamiento del otro. Desde una segunda mirada, se trata de co-elaboración de reglas de uso de las interacciones formativas propiamente tales.

No obstante, el " binomio » no se define para siempre, por ejemplo, un estudiante dado no tendrá necesariamente una supervisora en título durante el conjunto de su práctica. El binomio no está previsto que dure en el tiempo, ni se prevé por adelantado. Los nuevos serán tomados a cargo por distintos supervisores durante su periodo de acogida, y cada una de las supervisoras tendrá potencialmente a cargo una diversidad de nuevos miembros del equipo. Sin embargo, dos configuraciones se perfilan: los protagonistas se encuentran por la primera vez, o ya se conocían.

Deriva también del conjunto de situaciones observadas, la posibilidad de una aproximación cronológica en la elaboración de la relación, yendo desde el "descubrimiento del binomio ", hasta el momento en el que los protagonistas deben separarse (término de la fase oficial de " tutoría » o de " doblaje »).

\subsection{Combinar los saberes profesionales}

16 Abordar el aspecto de la combinación de saberes profesionales como último punto de la presentación del modelo, permite llamar la atención sobre el hecho que los elementos precedentes contribuyen a orientar la elección del tipo de contenidos tratados, la atención acordada a cada uno de ellos, o el énfasis con el que se subraya la importancia de tal o cual contenido. Esta postura no inhibe considerar que existen retroacciones sobre los dos otros componentes al momento de enunciar un contenido.

17 En nuestro análisis de los contenidos, adscribimos a la idea que los saberes profesionales tratados durante las interacciones formativas se encuentran "situados" en relación al contexto particular de trabajo (Cloutier et al., 2012). Sin embargo, incluso si es la acción en curso la que orienta los contenidos tratados, nuestros resultados 
muestran que estos pueden hacer referencia a espacios temporales pasados y futuros. Les comentarios que acompañan una indicación proporcionada por la supervisora pueden referirse a acciones anteriores, cuando los intercambios en el curso de las que se abordan las dimensiones de la actividad profesional con los estudiantes, par ejemplo, los llevan a proyectarse al futuro. La transmisión profesional es a la vez situada y diacrónica.

Siguiendo lo que han mostrado otras investigaciones sobre las situaciones de aprendizaje en el trabajo, nuestros resultados muestran también un amplio despliegue de las temáticas abordadas en el transcurso de las interacciones formativas. Estando interesada en las dimensiones específicas de la actividad de trabajo abordadas en el transcurso de los intercambios, los hemos clasificado en referencia a los cuatro polos alrededor de los cuales se organizan las actividades de servicio: el "sistema", los “ otros ", " si mismo ", y el " destinatario del servicio » (Caroly, \& Weill-Fassina, 2007), con el fin de insistir en el hecho que no se trata únicamente de saberes "técnicos". Nuestros resultados muestran también que los contenidos abordados durante las interacciones formativas, pueden centrarse sobre uno solo de los polos, o bien, los combinan directamente.

\section{La transmisión profesional : desarrollo de actividades reflexivas y revelador de las exigencias del trabajo}

19 Una idea que pone en evidencia este trabajo, es que no es posible definir de una manera simple lo que sería una "transmisión de calidad", pues no existen criterios formales simples para evaluarla. En todo caso, los análisis conducen a precisar dos cosas. Primero, una transmisión de calidad podría ser una transmisión donde el conjunto de componentes del modelo presentado podrían desplegarse. Segundo, una transmisión de calidad podría ser aquella donde se produjese el mayor número de actividades reflexivas, no sólo durante la transmisión, sino que igualmente en referencia a ella.

Desde nuestra perspectiva, el estudio de la transmisión en términos de interacciones formativas evidencia en si misma las exigencias de los sistemas de trabajo, sus variaciones y las posibilidades de enfrentarlas. ¿Podría pensarse que, mientras que una parte de las condiciones que hacen posible el mantenimiento de una elaboración de las interacciones formativas en situación de trabajo, sean ellas también las que permitan preservar la calidad del trabajo?

\section{BIBLIOGRAFÍA}

Caroly, S., \& Weill-Fassina, A. (2007). En quoi différentes approches de l'activité collective des relations de services interrogent la pluralité des modèles de l'activité en ergonomie ? @ctivités, 4(1), 85-98. http://www.activites.org/ v4n1/v4n1.pdf 
Cloutier, E., Fournier, P.-S., Ledoux, E., Gagnon, I., Beauvais, A., \& Vincent-Genod, C. (2012). La transmission des savoirs de métier et de prudence par les travailleurs expérimentés - Comment soutenir cette approche dynamique de formation dans les milieux de travail. Études et recherches, Rapport R-740. Montréal : IRSST.

Gheorghiu, M., Guillemot, D. \& Moatty, F. (2009). Changements organisationnels et mise en place de la tarification à l'activité dans les établissements hospitaliers français. Communication aux Journées internationales de sociologie du travail, juin, Nancy.

Molinié, A.-F., \& Volkoff, S. (2002). La démographie du travail, pour anticiper sur le vieillissement. Lyon : Editions ANACT.

Thébault, J. (2013). La transmission professionnelle : processus d'élaboration d'interactions formatives en situation de travail. Une recherche auprès de personnels soignants dans un Centre Hospitalier Universitaire. Thèse de doctorat en Ergonomie, CNAM/CEE-CREAPT, Paris.

Volkoff, S. (2011). Les éléments d'une nouvelle donne sociodémographique. In C. Gaudart et J. Thébault, Transmission des savoirs et mutualisation des pratiques en situation de travail, Actes du colloque de décembre 2009 (9-18). Cee, Rapport de Recherche, 64.

\section{AUTOR}

\section{JEANNE THÉBAULT}

Centre d'Etudes de l'emploi (Cee), Centre de Recherches sur l'Expérience, l'Age et les Populations au Travail (Creapt), “ Le Descartes I », 29 promenade Michel Simon, 93166 Noisy-le-Grand Cedex, France

jeanne.thebault@cee-recherche.fr 